\title{
Tangence
}

\section{Pour une phanéroscopie du spectacle}

\section{Louis Francœur}

Numéro 46, décembre 1994

Un théâtre de passage

URI : https://id.erudit.org/iderudit/025839ar

DOI : https://doi.org/10.7202/025839ar

Aller au sommaire du numéro

Éditeur(s)

Tangence

ISSN

0226-9554 (imprimé)

1710-0305 (numérique)

Découvrir la revue

Citer cet article

Francœur, L. (1994). Pour une phanéroscopie du spectacle. Tangence, (46),

38-53. https://doi.org/10.7202/025839ar d'utilisation que vous pouvez consulter en ligne.

https://apropos.erudit.org/fr/usagers/politique-dutilisation/ 


\section{Pour une phanéroscopie du spectacle Louis Francœur}

Le spectacle, que nous entendions par là les jeux enfantins que nous pouvons parfois observer dans la rue ou le jeu des acteurs que nous nous plaisons à contempler sur une scène de théâtre, peut être tenu pour un phanéron, c'est-à-dire pour un phénomène global au sens où la sémiotique ${ }^{1}$ entend ce terme.

La phanéroscopie du spectacle dont nous souhaitons poser les fondements dans cette étude, consisterait donc en l'analyse d'un type spécifique et particulièrement complexe de phanéron, celui que représente en effet le spectacle lorsque nous le définissons comme un "mégasigne" qui serait présent de manière continue à l'esprit des spectateurs de la rue ou de la scène tout le temps que dure la représentation. Du point de vue de la sémiotique, le spectacle de la rue ou de la scène serait susceptible d'entrer dans l'une ou l'autre des catégories suivantes: soit comme un réservoir de qualités susceptibles d'être déployées dans le temps et dans l'espace de la rue ou de la scène, soit comme une entité réelle tenant son existence dans son opposition à d'autres formes de représentations, soit comme une pensée créatrice globale qui s'inscrit à la fois dans le passé d'une tradition culturelle et dans l'avenir d'une culture à édifier. Ce sont les trois catégories que la sémotique nomme priméité, secondéité et tiercéité. Pour illustrer notre propos, nous retiendrons plus précisément deux types de spectacles, l'un appartenant aux jeux des enfants dans la rue, l'autre ayant trait à certains jeux scéniques de La trilogie des dragons de Robert Lepage.

L'observation (scopein) de ces deux phanérons nous amènera dans un premier temps à reconnaître dans toutes les formes de spectacle leur fondement "ground") c'est-à-dire, essentiellement, leur dimension ludique qui les constitue en spectacle. Par la suite, en nous fondant sur la fin qu'ils poursuivent ("goal") nous

1 Il s'agit en l'occurrence de la théorie des signes créee par le philosophe et homme de science américain Charles Sanders Peirce (1839-1914) qui préférait parler de phanéroscopie plutôt que de sémiotique. 
serons conduits à distinguer deux grandes classes de spectacles, la première concernera les spectacles ludiques proprement dits, la seconde aura trait particulièrement aux spectacles artistiques. Enfin, nous serons amenés à identifier les effets réels de chaque type de spectacle sur le spectateur. Dans le cas du spectacle ludique, nous pourrons observer que son effet réel réside essentiellement dans l'acquisition d'un savoir-faire particulier, tandis que lorsqu'il s'agit du spectacle artistique, son effet réel consiste plutôt dans le développement d'un savoir-être du spectateur artiste.

Ainsi présentée, la phanéroscopie du spectacle pourrait être entendue à la fois comme une théorie, comme une méthode d'analyse et comme une pragmatique.

Les études ethnologiques ont déjà abondamment traité de ce sujet particulier que représentent le jeu et le spectacle ludique dans les différentes cultures. Nous ne considérerons pas dans notre étude ce point de vue ethnologique illustré particulièrement dans le monde francophone par les travaux de Roger Caillois. Nous n'examinerons pas davantage l'apport des diverses sémiologies du spectacle qui, ces dernières années, ont multiplié les efforts pour tenter de rendre compte rigoureusement de cette réalité complexe qu'est le spectacle de la scène. Notre propos évitera systématiquement de discuter ces recherches et encore plus de soulever quelque controverse que ce soit, qui serait d'ailleurs aujourd'hui aussi vaine qu'inutile. Nous nous bornerons plus simplement à constater que jusqu'à présent, ce genre de sémiologie du spectacle s'est surtout attaché à procéder sytématiquement à la reconversion de la linguistique en sémiolinguistique dans l'espoir de rendre compte des phénomènes non verbaux. Faut-il nous étonner, alors, que nous soyons invités à passer de l'étude des textes linguistiques à celle des textes spectaculaires sans que vraiment la nature même des signes utilisés dans ces différents textes ait été véritablement interrogée? En revanche, le recours à la phanéroscopie, nous semble comporter de multiples avantages pour l'étude du spectacle comme forme spécifique de communication. Elle nous permettra, en premier lieu, de considérer tout spectacle comme un signe global, syncrétique, indécomposable autrement que dans ses trois parties essentielles que sont le représentamen, l'objet et l'interprétant dans la définition que donne du signe la sémiotique: «Un signe, ou représentamen, est quelque chose qui tient lieu pour quelqu'un de quelque chose sous quelque rapport ou à quelque titre. Il s'adresse à quelqu'un, c'est-à-dire qu'il crée 
40

dans l'esprit de cette personne un signe équivalent ou peut-être un signe plus développé. Ce signe qu'il crée, je l'appelle l'interprétant du premier signe. Ce signe tient lieu de quelque chose: de son objet. Il tient lieu de cet objet non sous tous rapports, mais par référence à une sorte d'idée que j'ai appelée quelquefois le fondement du representamen" ${ }^{2}$. Nous noterons particulièrement que ce signe triadique n'existe vraiment que par la présence et par l'action de son interprète, qu'il soit metteur en scène, acteur ou spectateur. Pas d'interprète des signes, pas de signes. Nous devrons alors pour chaque type de spectacle étudié, chercher et trouver ce qui, aux yeux de son interprète, metteur en scène, acteur ou spectateur le constitue comme signe dans une culture donnée. Ou, en termes sémiotiques, nous devons identifier quel est son fondement (ou son "ground"). Toutefois, une même représentation peut éventuellement posséder deux buts ("goals") différents qui la constituent comme deux megasignes ou deux spectacles différents: deux petites filles jouant avec des boîtes à chaussures vides, dans la rue ou sur la scène, poursuivent, en effet, deux buts différents, dont les conséquences sont de la plus haute importance pour les spectateurs. La complexité apparente de cette démarche sémiotique ne doit pas nous dissimuler le fait primordial que cette phanéroscopie du spectacle offre aux analystes du spectacle, pour la première fois sans doute dans la pensée occidentale, une théorie ${ }^{3}$ qui propose un seul système cohérent pour rendre compte des signes naturels et des signes culturels, des signes linguistiques et des signes non linguistiques, des signes digitaux et des signes analogiques, toutes espèces de signes confondues dans un même système sémiotique ${ }^{4}$. Enfin, la phanéroscopie placera au centre de l'analyse de chaque type de phanéron ou spectacle-signe, l'indispensable notion d'accroissement, non seulement l'accroissement des significations dans la multiplication des représentations mais aussi l'essentiel accroissement des habiletés ou du savoir-faire de l'interprète dans le cas du spectacle ludique, accroissement du savoir-être, développe-

2 Charles Sanders Peirce, Collected Papers, Charles Hartshorne and Paul Weiss (ed), Cambridge, Mass., The Belknap Press of Harvard University Press, 1965, vol II, par. 288. Nous adopterons dorénavant la notation convenue: C.P., 2.288 .

3 Voir la note précédente.

4 Marie Francoeur et Louis Francour, Grimoire de l'art, grammaire de l'être, Québec, Les Presses de l'université Laval et Paris, Klincksieck, 1993, p. 181-214. 
ment de l'être même de l'interprète, metteur en scène, acteur ou spectateur, dans le spectacle de la scène.

\section{Une théorie}

Que la phanéroscopie puisse constituer une théorie du spectacle, que celui-ci ait lieu dans la rue ou qu'il se produise sur la scène, nous n'avons pour nous en persuader qu'à porter attention à la définition même qu'elle propose du phanéron. Cette définition nous permet en effet de conférer à chaque élément signifiant du spectacle, qu'il soit linguistique ou non linguistique, qu'il s'agisse des dialogues ou de l'éclairage, des costumes ou de la gestuelle des acteurs, le même statut théorique de signe ou de phanéron. Toutefois pour saisir toute la portée de cette définition du phanéron, nous nous rappellerons que bien avant l'apparition de la sémiotique contemporaine, Aristote avait déjà classé les éléments de l'univers en dix catégories et que par la suite Kant en avait porté le nombre à douze. C'est en se fondant sur ces deux groupes de catégories que le sémioticien américain Charles Sanders Peirce en est arrivé à proposer que trois catégories seulement étaient nécessaires et suffisantes pour décrire tout phénomène observable, spirituel ou matériel, physique ou moral. Ces catégories sont celles que nous avons évoquées plus haut et qui ont trait à la possibilité qualitative, à l'existence réelle et à la pensée créatrice. Sera donc tenue pour un phanéron "la totalité collective de tout ce qui, de quelque manière et en quelque sens que ce soit, est présent à l'esprit, indépendamment du fait que cela corresponde à quelque chose de réel ou non "5. Pour éclairer la suite de notre propos, cette définition du phanéron appelle deux brefs commentaires. Si ce qui se déroule sous les yeux de quelque spectateur, dans la rue ou sur la scène, est susceptible d'être présent à son esprit, donc d'être un phanéron pour lui mais aussi pour tous les autres spectateurs qui l'entourent, cela signifique que chaque spectateur au moyen de ce premier phanéron sera en mesure d'appréhender quelque chose d'autre dont le premier tient lieu, son objet. Cet objet, qui est un second phanéron, doit être considéré comme tout aussi constitutif du spectacle que le premier. Enfin, ce qui permet de lier dans l'esprit des spectateurs ces deux phanérons, c'est la présence d'un troisième,

5 C.P., 1.284. 
42

distinct d'eux et qui rend présent à l'esprit de ces spectateurs les relations et les processus qui fondent les deux premiers dans leur entité respective de représentation et d'objet de la représentation $^{6}$. De ce point de vue, "il n'existe pas d'autre forme de conscience que les trois qui ont été mentionnées [...] Elles constituent une espèce de système " ${ }^{7}$. Le spectacle pourrait donc être tenu pour un système constitué de ces trois phanérons: un premier résidant dans ce qui est perceptible par les sens du spectateur ici et maintenant; un deuxième concernant ce qui est ainsi représenté et un troisième ayant trait à la relation qui rend possible la représentation de ce dernier par le premier. Chacun de ces phanérons est un signe à part entière qui peut être traité à son tour selon les trois catégories phanéroscopiques évoquées plus haut, celle de la priméité, celle de la secondéité et celle de la tiercéité, dont nous pouvons encore préciser que la première est le mode d'être de ce qui est tel qu'il est, positivement et sans référence à quoi que ce soit d'autre, que la seconde est le mode d'être de ce qui est tel qu'il est par rapport à un second, mais sans considération d'un troisième quel qu'il soit, et que la troisième est le mode d'être de ce qui est tel qu'il est en mettant en relation réciproque un second et un troisième ${ }^{8}$. Déjà le recours à ces catégories nous laisse entrevoir quelques conséquences pour l'analyse. Prenons le cas des boites à chaussures. Nous les remarquons, bien entendu, dans La trilogie des dragons de Robert Lepage où elles sont éminemment présentes, mais nous pouvons aussi les retrouver volontiers dans le souvenir de nos jeux d'enfants. Ces boîtes à chaussures, à quelque forme de spectacle qu'elles appartiennent, sont d'abord des objets réels perceptibles par tous; elles relèvent donc du premier phanéron, celui qui représente les choses. Il est cependant facile de concevoir que ce statut ne suffira pas à rendre compte de la réalité du spectacle auquel nous assistons, dans la rue ou sur la scène, spectacle pendant lequel ces boites à chaussures perdront leur entité autonome pour se voir investies de nouvelles significations chaque fois que, grâce, au troisième phanéron, elles seront mises en relation avec d'autres éléments formels du spectacle. Dans la pièce de Lepage, les boîtes à chaus-

6 Robert Marty, L'algèbre des signes. Essai de sémiotique scientifique d'après Charles Sanders Peirce, ed. A. Eschbach, Amsterdam/Philadelphia, John Benjamins, coll. "Foundations of Semiotics", no 24, 1989, p. 318 et s.

7 C.P., 7.551.

$8 \quad$ C.P., 8.328. 
sures constituent d'abord pour Françoise et Jeanne différents magasins puis, elles deviendront les objets essentiels du magasin de chaussures de Crawford à Toronto. C'est dire que le sens des phanérons est toujours posé d'abord comme une virtualité, qu'il est nécessairement téléologique et que sa signification n'est jamais complète, même une fois le spectacle terminé, du moins tant qu'il y aura des spectateurs susceptibles d'avoir ces phanérons présents à l'esprit et capables de les mettre en relation les uns avec les autres. C'est affirmer en même temps la présence continue et globale du système de relations qui existe nécessairement entre les éléments constitutifs du spectacle. De là à conclure qu'il nous est impossible d'isoler un élément du spectacle, comme par exemple de parler de signifiants puis par la suite de signifiés, il n'y a qu'un pas, que nous devons franchir. Car les éléments du spectacle que l'on isole volontiers, gestes, éclairage, bruits, objets, dialogues, indépendamment du fait qu'ils puissent posséder une individualité matérielle, sont, en fait, destinés à être présents à l'esprit d'un spectateur, ne serait-ce que l'acteur lui-même dans les jeux autistiques auxquels se livrent les enfants. À ce titre, ils relèvent d'une sémiosis triadique unique et indécomposable, à un moment unique du déroulement du spectacle. Cependant, qui dit déroulement, dit chaîne ininterrompue de phanérons. Chaque phanéron, à la manière de chaque acte de langage prenant place dans un discours linguistique, est en quelque sorte lié à celui qui le précède et à celui qui le suit par le télos, c'est-à-dire par l'orientation téléologique du spectacle. Nous pourrons alors décrire le déroulement du spectacle comme la succession des phanérons logiquement orientés vers leur fin ultime.

Toutefois, une seule et même chose, ces boîtes à chaussures par exemple dont nous parlions à l'instant, peut constituer plusieurs signes différents, tout dépendant des divers fondements ("grounds") du signe et des divers buts ("goals") poursuivis par l'interprète des signes, metteur en scène, acteur ou spectateur.

\section{Une méthode}

Le recours à la notion de fondement du signe ("ground") nous incite à considérer la phanéroscopie non seulement comme une théorie du spectacle mais tout aussi bien comme une méthode pour en analyser les diverses manifestations et leurs multiples formes. Nous rappellerons d'abord ce que dit du fonde- 
44

ment la définition du signe proposée plus haut: le signe tient lieu de son objet non sous tous rapports, mais par référence à une sorte d'idée appelée quelque fois le fondement du signe. Le fondement consiste donc en ce point de vue particulier en vertu duquel le signe pourra ou devra être interprété comme signe de son objet. Ainsi défini, le fondement peut être envisagé sous trois aspects différents et complémentaires : en tant que qualité qui peut être un signe (qualisigne), en tant que signe concret (sinsigne) ou en tant que signe qui est une loi (légisigne) ${ }^{9}$.

Quand nous observons les spectacles de la scène ou ceux des "jeux" enfantins de la rue, nous savons, ne serait-ce qu'implicitement, que tout dans leur manifestation n'est pas pertinent pour les définir comme signes culturels. Par exemple, nous pouvons noter que ce qui les constitue comme signes dans la culture, c'est-à-dire leur fondement pour les interprètes que nous sommes l'instant d'un regard furtif jeté sur les jeux des enfants ou sur ceux du théâtre, c'est leur commune caractéristique ludique.

Nous pouvons, en effet, noter l'inconstestable parenté qui existe entre le jeu et l'art ${ }^{10}$. Dans ses Lettres sur l'éducation esthétique de l'bomme, Friedrich Schiller avançait déjà l'idée que pour unifier les dimensions physique, logique et morale de notre être, nous devions parvenir à réconcilier dans notre nature deux forces également présentes, l'une représentée par le principe sensuel et l'autre par le principe rationnel. Puisqu'aucune de ces deux forces ne peut s'imposer pour parvenir à créer, seule, l'unité nécessaire à l'homme, Schiller suggérait que cette synthèse se réalise par le recours à un troisième principe, le principe ludique. Ce qui importe pour notre réflexion présente, c'est que nous prenions en compte que ce principe ludique, la sémiotique l'identifiera au principe esthétique.

9 David Savan, "La sémeiotique de Charles S. Peirce", Langages, $\mathrm{n}^{\circ}$ 58, 1980 , p. 9-23.

10 L'intérêt que porte Peirce à la dimension ludique des signes remonte à ses tout premiers écrits philosophiques, ceux qui constituent en réalité un commentaire des Lettres sur l'éducation estbétique de l'bomme de Friedrich Schiller. Bien que ce commentaire soit lui-même assez limité, Peirce n'en avoue pas moins que la lecture de ces Lettres de Schiller a produit en lui une telle impression qu'elles ont eu une influence sur son esprit qui s'est maintenue durant toute sa vie. Edward S. Petry Jr., "Peirce's Concept of SelfControl ", Transactions of the Charles S. Peirce Society, automne 1992, vol. XXVII, n० 4 , p. 669. 
La méthode que la phanéroscopie met de l'avant pour l'analyse des spectacles consiste essentiellement à envisager ceux-ci, quels qu'ils soient, selon leur fondement, c'est-à-dire selon le caractère particulier ou le point de vue en vertu duquel ils peuvent être interprétés comme des signes dans la culture. Ce point de vue ou fondement, c'est le principe ludique qui nous le fournit. Dans les jeux de la rue comme dans ceux de la scène, la réconciliation des principes sensuel et rationnel constitue la base même de leur existence. N'assistons-nous pas, en effet, à la substitution d'une situation réelle par une situation conventionnelle? Et, pourtant, comme spectateurs, ne sommes-nous pas témoins chez les enfants et chez les personnages de théâtre de comportements pratiques et conventionnels simultanés ${ }^{11}$ ? Cette bi-planéarité constitue le fondement ("ground") du signe-spectacle. Si maintenant nous recourons aux sous-classes du fondement que sont les qualisignes, les sinsignes et les légisignes, nous nous retrouvons avec une véritable méthode d'analyse des signes du spectacle. Voyons d'abord pour le qualisigne. Celui-ci est un fondement par sa seule qualité et indépendamment de toute relation spatiale ou temporelle à son objet. Par exemple, la qualité rectangulaire des boîtes à chaussures dans la pièce de Lepage qui permettra d'en faire des maisons et des magasins alignés dans la rue Saint-Joseph à Québec, qualité dont chacun a pu faire l'expérience, peut être tenue pour un qualisigne. Mais un qualisigne, en tant qu'il est un premier, n'est qu'une possibilité de signe. Il ne peut donner sa véritable mesure qu'une fois matérialisé dans un signe réel, qui est un second, appelé sinsigne. Gardant toujours à l'esprit notre référence aux boîtes de chaussures, l'alignement réel en forme de rue de ces boîtes, que ce soit dans les jeux de la rue ou dans ceux de la scène, constitue un élément important du fondement du signe-spectacle. Cependant les qualités, considérées pour elles-mêmes ou réalisées concrètement, n'auront de sens dans un spectacle que lorsqu'elles auront été en quelque sorte sanctionnées ou, mieux encore, lorsqu'elles seront établies dans une relation d'implication avec un troisième signe, le légisigne, qui est un signe-loi, une règle de conduite ou une convention. De telle sorte que nous pouvons nous représenter, mentalement ou graphiquement, cette relation d'implication qui va du légisigne

11 Voir à ce sujet, louvrage de Iouri Lotman, La structure du texte artistique, Paris, Gallimard, 1973, p. 99 et s. 
46

qui présuppose un sinsigne, lequel présuppose, à son tour, un qualisigne. La méthode d'analyse que nous propose ainsi la phanéroscopie se déroulera en trois temps: reconnaître en premier lieu à quelle classe de signes élémentaires nous avons affaire, des qualisignes, des sinsignes ou des légisignes, construire une représentation mentale ou graphique qui indique les relations d'implication existant entre ces signes et finalement fournir un signe globalisant qui les résume tous ${ }^{12}$. Dans l'exemple que nous avons utilisé jusqu'ici, nous pourrions formuler ce signe globalisant de la manière suivante: la mise en un certain ordre des boîtes à chaussures constitue un signe spectaculaire. Il va de soi que dans le cas particulier du spectacle théâtral, il faudra tenir compte du fait que nous sommes en présence d'une double convention ludique, une première concernant les rapports qui lient les personnages entre eux et une deuxième qui engage les spectateurs à l'égard du spectacle.

\section{Une pragmatique}

Cependant, si les spectacles de la rue et de la scène sont susceptibles de partager ce même fondement ludique, ils s'éloignent d'emblée l'un de l'autre pour ce qui regarde le but final ("goal") qu'ils poursuivent. Essentiellement, le but du jeu consiste, au moyen de l'acquisition d'habitudes et d'habiletés des joueurs, à conduire le spectateur à sanctionner positivement ou négativement l'acquisition et l'utilisation d'un savoir-faire, ce qui est le fait d'autres que lui-même. En revanche, le but de l'art vise à conduire le spectateur vers un savoir-être personnel nouveau. Loin de n'accorder de valeur qu'à la seule observation des règles, l'art aurait plutôt tendance à s'éloigner de la prescription dans la mesure où ce sont les procédés de désautomatisation des langages artistiques qui sont à l'origine de la vision nouvelle du monde qu'il entend proposer au spectateur. Éventuellement, le Moi artiste de chaque spectateur, qui ne peut être confondu avec le moi de l'artiste ou dans l'artiste ${ }^{13}$, en sera transformé.

Dans l'analyse que nous faisons des signes spectaculaires, nous devons en effet prendre aussi en compte les interprètes possibles de ces signes, notamment les interprètes ultimes que sont

12 Robert Marty, op. cit., p. 318 et s.

13 Marie Francœur et Louis Francœur, op. cit., p. 107. 
les spectateurs. Nous devons de plus connaitre les buts que ceuxci peuvent poursuivre en se présentant au spectacle, de même que tout ce qui pourrait justifier de leur part une certaine interprétation au regard de ce but ${ }^{14}$. Dans ce sens, la phanéroscopie est aussi une pragmatique puisque l'interprétation des spectacles se voit nécessairement orientée vers un savoir-être du spectateur. En effet, procéder à une description systématique d'un spectacle, c'est au point de départ s'assurer de l'interprétabilité des signes de ce spectacle, c'est-à-dire de leur possibilité de signification pour quelqu'un. L'interprétabilité d'un signe ne peut d'aucune manière être réduite à l'imposition d'un sens arbitraire et, en quelque sorte, extérieur au spectateur. Elle consiste plutôt dans le fait que le signe que le spectateur a sous les yeux est particulièrement apte à susciter un type particulier d'effet réel ou interprétant chez l'interprète qu'il est. Chaque signe du spectacle doit être investi de cette qualité essentielle d'interprétabilité. Cette possibilité d'interprétation n'a pas, cependant, à être toujours effectivement réalisée. Elle pourrait même éventuellement demeurer une pure possibilité. Ce qui, en revanche, apparaît d'une nécessité absolue, c'est l'orientation de toute cette activité d'interprétation vers une fin précise. Puisque aucun objet n'est un signe dans un spectacle qui ne soit pas interprétable et que toute interprétation effectuée par un spectateur est orientée vers une fin, aucun objet ne peut donc être considéré comme un signe s'il ne se trouve en relation avec une fin possible d'interprétation effectuée par un spectateur interprète. On aura compris que la phanéroscopie du spectateur-artiste que nous cherchons maintenant à cerner se situe d'emblée en marge de l'épistémologie qui a eu cours depuis le dix-neuvième siècle, notamment de celle qui est apparue naguère sous la forme du structuralisme. Nous ne pouvons, en effet, faire l'économie du sujet du savoir sous prétexte de saisir l'œuvre concernée dans toute sa "vérité" 15 , signifiant par là un désir irrépressible d'atteindre à l' objectivité" de la démarche de connaissance.

La phanéroscopie du spectacle nous permettra, pour sa part, d'observer le phénomène de croissance particulier dans lequel le

14 Thomas Short, "The Growth of Symbols", Cruzeiro Semiotico, $\mathrm{n}^{\circ}$ 8, Janeiro, 1988.

15 Michel de Certeau, Histoire et psychanalyse entre science et fiction, Paris, Gallimard, coll. "Folio", 1987, p. 92. 
48

spectateur d'une représentation artistique se voit engagé. L'expérience unique que celui-ci est appelé à vivre comme spectateur se confond en réalité avec l'expérience de la création même du spectacle artistique.

En définissant ce spectateur comme étant un signe, pour luimême d'abord mais également pour nous ensuite, les analystes des spectacles, en le désignant comme une forme de sémiosis ou signe-action, en le considérant comme un être doué d'une capacité singulière à se trouver en communication avec d'autres signes que sont les autres spectateurs, nous nous faisons en effet du spectateur une conception qui peut sembler n'avoir que peu d'affinité avec ce que la pensée cartésiennne nous a enseigné au sujet de l'être humain, centre du monde et maître incontestable de l'univers. Du reste, puisque chaque état de conscience du spectateur est le produit d'une inférence, le déroulement de sa vie créatrice au moment du spectacle sera essentiellement une suite d'inférences ou, si l'on préfère, un train de pensées et de sentiments mis en relation les uns avec les autres. Il s'ensuivra qu'à chaque instant de son existence de spectateur bic et nunc, celui-ci sera une authentique pensée et en tant que tel une espèce de symbole, c'est-à-dire un signe qui est pensée en vertu d'une convention ${ }^{16}$.

Si nous sommes fidèles à la sẻmiotique peircienne dans l'élaboration de la phanéroscopie du Moi artiste du spectateur, notre démarche s'opposera inévitablement à un certain nombre de propositions concernant des facultés que l'on prête à l'homme dans la tradition cartésienne. Par exemple, nous serons amenés à soutenir que nous ne possédons aucune possibilité de penser sans le concours des signes. Si, en effet, le spectateur artiste ne peut penser sans signe, il s'ensuit que lorsqu'il pense, il a présents dans sa conscience, des sentiments, des images, des concepts ou toutes autres formes de représentations qui agissent en lui à la manière des signes. Au reste, l'expérience qu'il a de sa propre existence, expérience souvent douloureuse puisqu'elle repose en grande partie sur l'ignorance et sur l'erreur auxquelles il ne peut échapper, est susceptible de l'amener à croire que tout ce qui est présent à sa conscience n'est qu'une manifestation phénoménale ou phanéroscopique de lui-même. De telle sorte que lorsqu'il pense, 
il se découvre lui-même comme un signe. Son Moi, dans ce cas, est pour lui-même un signe. Il est un signe pour une pensée qui l'interprète, la sienne propre; il est un signe pour un objet pour lequel, dans cette pensée, il est l'équivalent; il est un signe qui, sous quelque rapport ou quelque qualité, établit un lien avec son objet ${ }^{17}$. C'est dans l'exploration méthodique de cette conception anticartésienne du Moi développée par la sémiotique que nous sommes le plus susceptible de trouver les éléments constitutifs de la phanéroscopie du Moi artiste du spectateur.

Nous proposerons, en effet, d'envisager ce dernier, comme toute espèce de signe, selon les trois catégories sémiotiques de la priméité, de la secondéité et de la tiercéité. Dans cette démarche théorique, nous nous abstiendrons de tenir la dimension sémiotique pour une donnée psychologique ${ }^{18}$, de même que nous nous garderons de confondre les vérités psychiques avec les vérités psychologiques ${ }^{19}$.

La conscience que le Moi artiste du spectateur a de lui-même dans l'immédiateté est un sentiment ("feeling") unique de ce que sera sa personnalité unique, grâce à ce spectacle. C'est là la dimension de priméité de son Moi. "Premier, est la conception de l'être ou de l'exister indépendamment de toute autre chose "20. Non seulement chaque spectateur artiste possède-t-il une conscience aiguë du caractère qui lui est propre, mais le sentiment de "posséder per se différentes qualités" 21 lui permet d'affirmer sa différence à l'endroit de tous les autres spectateurs. En effet, puisque, par définition, cette qualité de sentiment est unique, elle ne pourra d'aucune manière être confondue avec les qualités et les sentiments des autres spectateurs artistes qui, eux aussi, et pour les mêmes raisons, sont uniques. Ce statut établit le spectateur comme le lieu de tous les commencements, de tous les possibles, de toutes les virtualités de signification. Nous pourrions affirmer du Moi artiste du spectateur, dans sa priméité, qu'il sera jugé à ses fruits, c'est-à-dire dans le futur seulement ${ }^{22}$. Cette référence au futur est un élément essentiel de sa personnalité. Si, en effet,

17 C.P., 5.283.

18 Peirce, 8.330. Lettre à Lady Welby du 12 octobre 1904.

19 C.P., 8.330 et 5.485 .

20 C.P., 6.32.

21 C.P., 3.568.

22 C.P., 5.402, note 2 . 
les fins ultimes de ce spectateur étaient déjà connues de lui, explicites pour tous, il n'y aurait aucune place chez lui pour le développement, pour l'accroissement, bref, pour la vie. Conséquemment, il n'y aurait pas de personnalité ${ }^{23}$. Lorsque nous tenons de tels propos, nous laissons entendre par là même que le Moi artiste du spectateur en tant que premier constitue seulement une entité inchoative, un germe en quelque sorte, délibérément et entièrement tourné vers l'avenir du spectacle, toujours en voie d'accomplissement, jamais achevé. Dans notre élaboration de la phanéroscopie du Moi artiste du spectateur, nous devons prévoir que celui-ci devra toujours être envisagé, en premier lieu, comme un être "présent et immédiat [...], frais et nouveau,[...] inchoatif, original, spontané et libre[...], vif et conscient[...]"24.

C'est pourquoi, dans cette démarche, assurons-nous une place de toute première importance à la dimension de secondéité du signe, si nous voulons saisir le Moi artiste du spectateur dans son existence. Nous n'entendons pas signifier ici l'existence que l'on confond généralement avec la vie quotidienne, mais plutôt celle qui appartient à un ordre différent, celui du monde artistique. En revanche, nous aurions tort de croire que le Moi artiste du spectateur nous sera facilement accessible, comme le sont les personnages familiers de notre vie quotidienne. À la vérité, nous n'atteindrons à la véritable connaissance du Moi artiste du spectateur qu'à la lumière de ses réactions, voire de ses oppositions, à l'intérieur du monde artistique théâtral. "Second, est la conception de l'être dans sa relation à quelque chose d'autre "25. Dans sa dimension de secondéité, le Moi artiste du spectateur nous sera dévoilé, en somme, autant par les limites que lui impose son appartenance au monde de l'art en ce moment même que par l'affirmation de son altérité à l'endroit de ceux qui partagent sa condition de spectateur.

"L'existence est ce mode d'être qui réside dans l'opposition à un autre. [...] Une chose sans opposition ipso facto n'existe pas "26. Cette expérience, essentielle pour le spectateur, s'accomplit dans le choc de ce qui à ses propres yeux est tenu pour artistique et de ce qui ne l'est pas. Nous sommes donc conviés à

$\begin{array}{ll}23 & \text { C.P., } 6.157 \\ 24 & \text { C.P., } 1.357 \\ 25 & \text { C.P., } 6.32 \\ 26 & \text { C.P., } 1.457\end{array}$


reconnaître une individualité, celle du Moi artiste du spectateur du jeu théâtral, dont l'essence même consiste à réagir à l'autre et à prendre ainsi conscience de sa propre existence de spectateur ${ }^{27}$. Une existence, en fait, qui repose sur la double conscience qu'il nourrit en lui, celle de l'effort qu'il doit déployer pour venir au monde artistique tout au long de la manfestation théâtrale et celle, non moins douloureuse, de la résistance qu'il rencontre pour demeurer dans ce monde auquel il vient d'accéder, le temps de la représentation et, dans plusieurs cas, après celle$\mathrm{ci}^{28}$. L'entrée dans le monde artistique entraîne une rupture radicale de la relation que cet homme ou cette femme qui devient spectateur ou spectatrice artiste entretenait avec son monde quotidien. L'existence du Moi artiste du spectateur le condamne à se situer d'emblée dans l'autrement organisé. Dans ce cas, l'être du Moi artiste du spectateur a bien peu à voir avec le réel, sauf ce qu'il lui emprunte pour créer son propre univers artistique.

Pourtant, si le Moi artiste du spectateur est un signe dont nous pouvons, grâce à la dimension de secondéité de la phanéroscopie, saisir l'existence dans les limites de son univers même qui est celui de l'art, cet aspect est loin de nous donner accès à tout ce que nous avions entrevu dans le Moi premier comme possibilité d'accomplissement, à tout ce qui existait en germe et qui était susceptible de s'épanouir. C'est grâce à la tiercéité de la phanéroscopie, que nous pourrons à loisir observer le déroulement du fil de sa vie ${ }^{29}$, savourer, en somme, les fruits dont il était la promesse. "Troisième est la conception de la médiation, ce par quoi un premier et un second sont mis en relation.30. En tant que troisième, le Moi artiste du spectateur sera un signe qui est pensée, pensée de la relation qui existe entre ce qui n'était au départ que virtualité et ce qui existe réellement bic et nunc sur la scène et dans la salle, pensée de la relation nécessaire entre son passé, son présent et son avenir évoqués ou représentés sur la scène, pensée souveraine et dépendante, autonome et issue d'un train de pensées engendré par le spectacle lui-même. Étroitement relié à l'ensemble des spectateurs présents autour de lui, le Moi artiste du spectateur n'est plus, alors, seulement ce Moi limité que 
52

nous avons identifié auparavant comme autre et différent, il se présente maintenant comme faisant partie de la conscience artistique universelle partagée par tous ceux qui sont dans la même situation que lui, comme un signe vivant parmi une foule d'autres signes vivants qui l'ont précédé ou accompagné dans l'univers de l'art théâtral. En effet, comme le spectacle qu'il contemple est issu d'autres spectacles, ainsi le Moi artiste du spectateur trouve-t-il sa place dans la longue procession des spectateurs qui l'ont précédé, autres Moi artistes. Si, dans sa dimension tertiaire, nous concevons le Moi artiste du spectateur comme étant nécessairement situé en continuité avec les autres spectateurs artistes, nous entendons par là qu'il s'agit avant tout d'une famille de pensée. En effet, le Moi artiste du spectateur ne devient signe authentique et complet dans la culture que par sa participation à la pensée commune qui le constitue lui-même comme pensée, donc comme signe.

Le Moi artiste du spectateur n'atteindra ainsi sa pleine personnalité que dans son intégration à la conscience collective illimitée et indivise de tous les spectateurs, d'abord, mais aussi à la conscience de tous les interprètes du monde de l'art, qui s'est formée depuis que l'homme crée et découvre des signes et des formes, et les interprète. Il n'en va pas autrement du spectateur que de l'être humain en général, qui ne peut prétendre être complet aussi longtemps qu'il demeure seul, car il est essentiellement, dès sa naissance même, un membre virtuel de la société, son expérience n'ayant aucune valeur s'il demeure isolé ${ }^{31}$. Aussi longtemps que le Moi artiste du spectateur était considéré hors de la catégorie de la relation et de la pensée, nous étions fondés de le décrire comme un signe incomplet; c'est la dimension de tiercéité qui lui confère la totalité de son être. Cette dimension de la phanéroscopie ne laisse pas de nous rappeler que la description que nous faisons du Moi artiste du spectateur doit prendre en compte sa relation nécessaire à un temps et à un espace déterminés. D'une part, en effet, il appartient totalement à son temps avec les conséquences sérieuses que cette affirmation, en apparence banale, implique: être de son temps, pour un spectateur, c'est d'une certaine manière devoir assumer le passé, plus spécifiquement tout le passé théâtral de son temps. D'autre part, le Moi artiste du spectateur vit une expérience culturelle dans une collectivité don- 
née. Quel que soit son degré de conformité ou d'opposition aux valeurs esthétiques de cette collectivité, il est responsable, au même titre que tous les autres artistes, par son appartenance fraternelle même à cette collectivité, de son développement continu. Loin d'être solitaire, le Moi artiste du spectateur est, par nature, un être communicable ${ }^{32}$. Cette dimension sémiotique du Moi artiste du spectateur le pousse à participer à une communication déjà existante dans laquelle il vient tout naturellement s'inscrire. Il s'agit, en effet, d'une des notions fondamentales de la phanéroscopie, que cette tendance irrépressible du Moi artiste du spectateur à se répandre continuellement, comme s'il était porteur d'un dynamisme intrinsèque qui le pousserait, sans qu'il puisse y avoir intervention de facteurs déviants, à s'intégrer à des ensembles d'une complexité toujours plus grande ${ }^{33}$. En se répandant ainsi le Moi artiste du spectateur est peut-être susceptible de perdre de son acuité, de son intensité, mais de façon générale il ne pourra que sortir gagnant de cette expansion en devenant indissolublement lié aux autres ${ }^{34}$. Car tous ces Moi artistes de spectateurs, réunis par une véritable communion au sein d'un même continuum de pensées au cours d'un même spectacle, sont convoqués à former un Moi d'un ordre supérieur que nous identifions à la culture. Ceci ne devrait pas nous surprendre outre mesure si nous nous souvenons que la signification ultime d'un spectacle se dêveloppe toujours sous la forme d'un dialogue entre les différents Moi artistes des spectateurs 35 .

Ainsi décrite, la phanéroscopie du spectacle lie indissociablement les unes aux autres les dimensions théorique, méthodologique et pragmatique de l'univers du spectacle. 